\title{
Characterization of Kevlar Using Raman Spectroscopy
}

\author{
Glenn Washer, Ph.D., P.E. ${ }^{1}$, Thomas Brooks ${ }^{1}$ and Regor Saulsberry, P.E. ${ }^{2}$ \\ ${ }^{1}$ Department of Civil and Environmental Engineering \\ University of Missouri-Columbia \\ 2503 Lafferre Hall \\ Columbia, MO 65211 \\ ${ }^{2}$ Project Manager \\ NASA - White Sands Test Facility \\ Las Cruces, NM 88012
}

\section{Abstract}

This paper explores the characterization of Kevlar composite materials using Raman spectroscopy. The goal of the research is to develop and understand the Raman spectrum of Kevlar materials to provide a foundation for the development of nondestructive evaluation (NDE) technologies based on the interaction of laser light with the polymer Kevlar. The paper discusses the fundamental aspects of experimental characterization of the spectrum of Kevlar, including the effects of incident wavelength, polarization and laser power. The effects of environmental exposure of Kevlar materials on certain characteristics of its Raman spectrum are explored, as well as the effects of applied stress. This data may provide a foundation for the development of NDE technologies intended to detect the in-situ deterioration of Kevlar materials used for engineering applications that can later be extended to other materials such as carbon fiber composites.

\section{Introduction}

The use of polymer composite materials for the design and construction of engineered components has increased steadily since Kevlar was first introduced by the Du Pont de Nemours Inc. in 1971. The materials are formed by a combination of high-strength fibers and polymer 
matrix resins that exhibit the composite mechanical properties of the materials used. The materials have been used to replace a wide variety of metallic components in aerospace and military structures, capitalizing on the high strength-to-weight ratio of the materials to realize mass reductions in engineered components [1]. For civil engineering applications, composite materials are increasingly used to strengthen structures and provide increased durability, benefiting from both the formability of the materials in the field and the high strength-to-weight ratio [2]. For example, in the state of Texas, it is common practice to repair bridges that have been damaged from collision with over-height vehicles by patching the concrete with a carbon fiber/epoxy composite. Other State Departments of Transportation are using these materials to improve the seismic resistance of bridges, and to rehabilitate aging concrete structures [2].

The increasing use of these materials has introduced a need for the development of methods to evaluate the condition of polymer composites during their service lives. This has led to the development of a variety of nondestructive evaluation (NDE) technologies that are intended to be used to evaluate the condition of polymer composite materials in-situ. This has included a variety of acoustic/ultrasonic techniques, thermographic and electromagnetic methods that are intended to detect damage to the composite $[3,4]$. In general, these methods are intended to detect mechanical damage in the materials caused by impact or overstress of the materials. The effect of the environment on material properties has remained largely unexplored, due in part to the complex interaction of composite materials with their environment. For example, polymer composites typically exhibit a failure mode of creep rupture, a reduction in the mechanical capacity of the material as a result of exposure to elevated stresses over time [5]. Additionally, degradation of the fiber/matrix binding may result from water absorption and other environmental effects, leading to a reduction in composite action between the fibers and the matrix that provide the material its strength and durability [5]. For Kevlar, exposure to ultra-violet (UV) radiation can also damage the fibers by altering its chemical structure [6]. 
Laser Raman spectroscopy is a light scattering technique that is widely known as a tool for chemical characterization. This technique has been traditionally applied in the laboratory by chemist, physicists and material scientists as means of determining the chemical structure of materials. The technique generally involves analyzing a spectrum of scatter light that exhibits certain peaks in light intensity that relate to the chemical structure of the tested material. For Kevlar, preliminary experimental testing indicated that there are as many as 16 separate, identifiable peaks in the Raman spectra of Kevlar, depending on the incident laser wavelength used to initiate the scattering. Each of these peaks is associated with a vibrational mode of the polymer that are generally effected by applied strain and molecular structure and bonding. These effects have been successfully applied to evaluation of the stress and strain distribution of composite materials, where shear stresses transfer load between individual fibers through the matrix resin [6-13]. The research reported here explores the use the Raman technique to identify environmental deterioration of Kevlar materials. The effects on certain characteristics of the Raman spectra resulting from prolonged exposure to stress at elevated temperatures are discussed, as well as the effects of applied elastic stresses. Experimental issues related to evaluating the Raman spectra of Kevlar are also discussed, including the effect of fiber orientation and laser power levels on the effective acquisition of the spectra. Fundamental data is provided that could lead to the development of NDE techniques for the in-situ condition assessment and materials characterization of composite materials.

\section{Background}

Raman spectroscopy can be used to study the chemical composition and structure of polymer materials such as Kevlar. This method involves illuminating the surface of a material with an incident laser light and observing the scattering characteristics. When an incident photon from the laser interacts with a molecule, the photon can be absorbed, reflected or scattered. Scattering may be elastic, known as Rayleigh scattering, or inelastic Raman scattering. For the Rayleigh scattering process, the energy of the incident photon is equal to the emitted or scattered photon. Raman spectroscopy is the measurement of the intensity and frequency of the 
inelastically scattered light. Raman scattered photons are shifted from the incident energy due to change from the vibrational energy of the molecule illustrated in Figure 1. The photon may lose energy (Stokes scattering) from the incident photon depositing energy in the molecule or gain energy (anti-Stokes scattering) due to the loss in energy of the molecule. In the case of Stokes scattering, the photon will decrease in frequency and increase in wavelength. Stokes scattering is typically used for analysis due to higher intensities when compared to anti-Stokes scattering [14].

To produce a Raman spectrum, a laser provides incident light upon the surface of a sample, and light is back-scattered over $180^{\circ}$ and collected through optical lenses. Generally, for rigid chain polymer analyses, the range of wavelengths of the incident laser is from $488 \mathrm{~nm}$, which is visible, blue light to $1064 \mathrm{~nm}$, near infrared light. The inelastically scattered light is then guided through a monochromator and dispersed onto a charge coupled device (CCD). For a Raman spectrometer system, this process is shown on a schematic diagram in Figure 2. The CCD output is the intensity of scattered light per wavelength, which is converted to a wavenumber and subtracted from the wavenumber of the incident beam to create a spectrum of the shifted, Raman-scattered light. Figure 3 illustrates how a Raman spectrum is developed from Stokes scattering. From the figure, the abscissa is the absolute wavenumber of the scattered light resulting from an incident laser wavelength of $647.1 \mathrm{~nm}$. A second abscissa shown indicates the wavelength and relative wavenumber for the frequency shift from the incident laser light. The wavenumber is simply the inverse of the wavelength. The ordinate represents the relative intensity of the scattered photons per second, typically detected by a charge coupled device $(C C D)$ or photomultiplier tube. As shown in the figure, the absolute wavenumber of inelastic scattered light, $\bar{v}_{1}$, is subtracted from the absolute wavenumber of incident light, $\bar{v}_{o}$, to calculate a relative wavenumber to the incident light, $\bar{v}_{r}$. Therefore, the Stokes scattering equation becomes $\overline{v_{r}}=\overline{v_{o}}-\overline{v_{1}}$. 
The frequency shifts of the vibrational energy that occur in a Raman scattering process are independent of the incident photon frequency but dependent upon the specific molecular geometry of the material. For frequency shifts to occur such that certain vibrational modes are Raman active, the polarizability of the molecules must change during vibration. The polarization of the molecule is dependent upon the symmetry of the molecular vibration for a specific scattering geometry [15]. A certain material of molecular structure could have many shifts in frequency of the incident photons, revealing a Raman spectrum. For the molecular structure of Kevlar, shown in Figure 4, a series of aromatic rings are bonded through oxygen and hydrogen bonds in the monomer. As a result, the $\mathrm{C}-\mathrm{C}$ aromatic ring has certain vibrational modes (e.g. stretching, axial extension) as does the $\mathrm{H}-\mathrm{O}$ and $\mathrm{H}-\mathrm{C}$ bonds. Many of the vibrational modes associated with these bonds are Raman active, such that Kevlar material produces a complex and rich Raman spectrum. Changes in the bonding conditions, such as changes in interatomic forces that might result from applied strain, create shifts in the wavenumber of peaks in the spectrum [12].

A Raman spectrum of Kevlar yarn is presented in Figure 5. The abscissa is the wavenumber for the frequency shift from the incident laser light, over a range of 600 to $1700 \mathrm{~cm}^{-1}$. The ordinate represents the relative intensity of the scattered photons (laser light). A number of intense peaks, or bands, are shown in the spectrum, each corresponding to a vibrational mode of the polymer material. Analytical models have been developed that predict these peaks; for example; the peak at $\sim 1613 \mathrm{~cm}^{-1}$ stems from a stretching vibrational mode of the C-C phenyl ring, while the 1649 $\mathrm{cm}^{-1}$ peak stems from the stretching of the $\mathrm{C}=\mathrm{O}$ bond (Figure 5). Raman scattering analyses of Kevlar have determined a series of well defined peaks ranging from 600 to $1700 \mathrm{~cm}^{-1}$ as shown in Table $1[16,17]$. From these analytical models, specific peaks identified in the spectra can be related to the structural configuration of the material.

Since Kevlar has a well-defined Raman response, there have been many studies performed that characterize the structure of the polymer material and evaluate the material under applied 
strain/stress $[7-12,16]$. The majority of previous studies on the material have analyzed the $\sim 1613 \mathrm{~cm}^{-1}$ peak which is produced from the C-C stretching of the phenyl ring due to the high sensitivity of the peak shift under applied strain/stress, high intensity relative to other peaks in the spectrum, and relevance to the fundamental structure of the polymer.

The relationship between the position of peaks in the spectrum and applied stress/strain has been investigated by many researchers. Galiotis et al. [9] recorded Raman spectra of strained Kevlar 49 fibers embedded in a matrix epoxy. A $514 \mathrm{~nm}$ argon ion laser operated at $5 \mathrm{~mW}$ with the laser light polarized parallel to the fiber and polymer chain axis was used. It was observed that the whole Raman spectrum between 1100 and $1700 \mathrm{~cm}^{-1}$ (six Raman bands) shifted to lower wavenumber with applied tensile strain. Each of these peaks shifted at a different rate as a function of applied tensile stress. It was also reported that broadening of the Raman bands was observed. Both peak shifting and band broadening indicated that the $\sim 1613 \mathrm{~cm}^{-1}$ Raman band exhibited the highest sensitivity. Prasad and Grubb [6] found a decrease in wavenumber and an increase in the Raman band width with applied tensile stress for Kevlar 49. At a molecular level, when the fiber is stressed, changes occur in bond length and bond angle which could be observed on the Raman spectrum as shifts in peak frequency. The band broadening is created from defects in the fiber or defects being created in the fiber under stress. Furthermore, the load is transferred by secondary hydrogen bonds between the long chains of molecules. If there are defects present, the resulting stress redistribution creates a greater increase in band width. The hydrogen bonds referred to are shown as dashed lines in Figure 4. It was also reported the number of defects in a Kevlar fiber was increased by exposure to ultra violet light, and an increase in band broadening under applied load was observed [6]. Other investigators have explored the effect of water immersion on the Raman spectra of Kevlar [10, 18]. Kawagoe et al. [10] applied tensile stress to a dried sample of Kevlar and to samples that had been immersed in water for a 24 hour period. The rate of peak shifts as a function of applied loads appeared the same in both samples. Another study by Stuart [18] examined the Raman spectra of Kevlar that had been immersed in water. The intensity of bands appearing in the spectra relative to the 
$\sim 1278 \mathrm{~cm}^{-1}$ band, rather than the position of peaks (shifting), was analyzed to determine if water immersion was effecting the bonding within the Kevlar. A decrease in intensity of the $\sim 1613 \mathrm{~cm}^{-1}$ band was found for the samples immersed in water when compared to samples that had not been immersed. The loss of intensity for this band was attributed to the disruption of hydrogen bonding in the Kevlar as a result of immersion in water.

These previous investigations indicate the spectrum characteristics such as peak position, bandwidth and relative peak intensity can be related to external conditions such as applied load and environmental exposures. This suggests that changes in the material on a molecular level that result from damage induced by aging, i.e. exposure to environmental loadings such as stress, temperature, moisture, etc., may be detectable by examining the characteristics of Raman spectra. As such, the technique may provide the foundation for an NDE approach that examines materials in-situ for purpose of detecting deterioration. The effects on characteristics of the Raman spectra resulting from exposure to elevated temperature and stress are examined here to evaluate the potential of the method as a tool for nondestructive evaluation of the material. Such a method could potentially be extended to other polymer-based composites.

\section{Kevlar}

Kevlar fiber reinforced composite material is a strong, lightweight material consisting of high modulus Kevlar yarn embedded in a matrix; the combined system is referred to as a fiber reinforced polymer (FRP). Kevlar fibers consist of long molecular chains produced from a synthetic polyamide in which the amide linkages are attached directly between two aromatic rings. An amide linkage is an organic functional group characterized by a carbonyl group, $\mathrm{C}=\mathrm{O}$, linked to a nitrogen atom, and an aromatic ring is a molecular structure consisting of six carbon atoms connected by alternating single and double covalent bonds. These long chains are highly oriented with many interchain hydrogen bonds and aromatic stacking interaction resulting in high energy absorption. As a result, Kevlar 49 fibers have a high tensile strength-to-weight ratio. The 
low-density material also has a high modulus-to-weight ratio; ideal for engineering composites. The fibers (yarn) have an elastic modulus of $120 \mathrm{MPa}$, a diameter of $12 \mu \mathrm{m}$ and an elongation of 2.8\% [19]. Kevlar fibers have a low compressive strength and the mechanical properties vary with temperature. The maximum temperature for Kevlar fiber usage is $250^{\circ} \mathrm{C}$ due to the polymer decomposing at higher temperatures [19]. Kevlar fibers are embedded in a matrix of epoxy to form composite strands suitable for engineering applications. The Kevlar strands are affected by visco-elastic deformation under sustained loading i.e. creep and degradation as a result of exposure to ultraviolet light, elevated temperature and water.

\section{Experimental}

\section{Materials}

A series of Kevlar samples have been evaluated to identify and characterize the Raman spectra. This has included Kevlar yarn that is comprised solely of the Kevlar fibers in a tow, without matrix materials such as epoxy to bind the individual fibers together into a composite material (1140 denier). The yarn material was tested to characterize the spectra of the Kevlar fibers without epoxy, to provide baseline spectra for comparison and to develop methods for yielding spectra in later tests on strand samples.

Strands of Kevlar consisting of a yarn tow impregnated in an epoxy matrix to form a composite have also been tested. The strand specimens tested included "virgin" strands that have not been exposed to any type of environmental loading, such as high temperature and stress. Virgin samples of Kevlar yarn and composite strands consisting of Kevlar fibers impregnated in an epoxy matrix were obtained from Texas Research International (TRI) for testing. Virgin strands were used for direct comparison to strands that were "aged" by exposure to elevated temperature and elevated stress. Among these aged strands, a series of strands that had been part of a creep testing program were evaluated. These strands were loaded to $65 \%$ of their tensile 
strength and held at load for various periods of time at different temperatures. Strands exposed to this testing program are referred to as "creep" specimens. A virgin strand of the same materials was used for comparison with the creep samples.

Another set of strands specimens labeled were evaluated as part of the study. These strands were exposed to accelerated aging tests that attempted to determine the potential stress rupture lifetime of the materials. The SIM (stepped isothermal method) specimens were loaded to $65 \%$ of its tensile strength in a temperature-controlled chamber. Step-wise increases in the temperature were imposed at sustained stress levels until failure [20].

In addition to the strand specimens, two sections of composite materials were also evaluated. The composite sections were removed from aged composite over-wrapped pressure vessels (COPVs) that were part of a NASA fleet leader program. The fleet leader program maintained a population of COPVs under pressure for extended periods of time to provide data on lifetime performance characteristics of in-service COPVs of similar design. One of these specimens, SN 032, was aged for $\sim 17$ years at $80^{\circ} \mathrm{C}$ while pressurized at $29 \mathrm{MPa}$. A second composite specimen, SN 007, was cut from a fleet leader COPV that had been aged at a pressure of 29 MPa for 17 years at room temperature. Each of these fleet leader specimens were sectioned into two pieces ( $a$ and $b)$ for comparison.

\section{Instrumentation}

The Raman spectra reported here were measured using two separate instruments. A Renishaw micro-Raman system was used to measure Kevlar 49 using an incident laser of $488 \mathrm{~nm}, 647 \mathrm{~nm}$ and $752 \mathrm{~nm}$. The multi-frequency spectrometer enables the study of wavelength effects that can alter the appearance of the Raman spectra. The ability to evaluate the quality of the spectra obtained over a range of frequency, e.g. wavelengths, allows for the evaluation of the optimum frequency for experimental study. The laser source illuminates the sample surface through a $50 \mathrm{x}$ microscope objective. Raman scattered light is collected from the surface and a spectrometer 
with a CCD detector is used to evaluate the intensity of the Raman scattered light over a range of wavelengths.

The collection time was generally averaged over two to three scans for 30 seconds each with a pixel resolution of $\sim 1.6 \mathrm{~cm}^{-1}$. Power was kept under $10 \mathrm{~mW}$ and the incident light was polarized parallel to the fiber axis. The spectral region was tested from 600 to $1700 \mathrm{~cm}^{-1}$.

Raman measurements were also taken using a Fourier Transform (FT) Raman system with a near-infrared incident laser of $1064 \mathrm{~nm}$. The FT Raman system differs from the spectroscopic system in the determination of the wavelength of scattered light, which is determined by an interferometer rather than by a spectrometer. An interferometer is used due to the inefficient gratings and throughput-limited slit configuration of spectrometers at longer wavelengths [21]. The interferometer has the ability to remove the Rayleigh scattering in the near-infrared range. FT Raman systems use of the $1064 \mathrm{~nm}$ laser removes fluorescence completely. In the nearinfrared (IR) region of $1064 \mathrm{~nm}$, the photon energy is usually not sufficient to cause transitions between electronic states that give rise to fluorescence [22]. For each spectrum, 32 scans were Fourier transformed with a resolution of $1.5 \mathrm{~cm}^{-1}$. All spectra were analyzed using the commercial software Peakfit@.

\section{Effects of Fiber Orientation}

The individual monomers of Kevlar 49 are highly oriented within the material fibers, generally at a $10^{\circ}$ angle from axial along the fiber length [6]. This is the primary reasons the material exhibits such highly anisotropic elastic behavior, with very high axial tensile strength and nominal compression strength. As a result of the orientation of the molecules within the polymer, the polarizability of the material is highly dependent on its orientation relative to the polarization of incident light. Testing was conducted to ensure the proper alignment of specimen in the microRaman system to ensure the most intense Raman spectra from the material. A Kevlar strand was mounted to a rotating device and scanned with the micro-Raman system from 0 to $360^{\circ}$ in 
$10^{\circ}$ intervals. Figure 6 displays two views of a Kevlar strand rotated at different angles under the Raman microscope. Figure 7 illustrates the differences in appearance of the spectrum when the laser is polarized parallel to the fiber axis and perpendicular to the fiber axis. Raman peaks are nearly indistinguishable from the background noise when the laser is polarized perpendicular to the fiber axis.

Figure 6 also shows the texture of the surface of a Kevlar strand as seen through the $50 \mathrm{X}$ objective. As shown in the figure, the surface of the strand material is characterized by a series of alternating fiber and matrix bands at the surface. These alternating surface textures have an effect on intensity of the Raman spectra created, as discussed later in the next section entitled Laser Power.

\section{Laser power}

Problems arise when too much laser power is incident upon Kevlar samples. Literature indicates that the power of the incident laser may damage Kevlar fibers if above $\sim 16 \mathrm{~mW}[6,16]$. Galiotis et al. [23] also studied the effects of incident power levels by increasing the power up to $20 \mathrm{~mW}$ for a set of scans while straining a Kevlar 49 fiber at $2.5 \%$. The power was then decreased which resulted in a significant shift to a higher wavenumber of the $\sim 1610 \mathrm{~cm}^{-1}$ peak, corresponding to a strain relaxation of $1.48 \%$. These results indicated that the fibers had been damaged as a result of being exposed to the higher incident laser power. In this study, the power levels have been closely controlled and kept at less than $10 \mathrm{~mW}$ on the sample surface.

The lowest power that provided a suitable spectrum was determined experimentally using a virgin strand of Kevlar 49. Figure 8 shows spectra at different power levels. From the figure it is shown that a power level of $7 \mathrm{~mW}$ is adequate for providing a spectrum with high $\mathrm{S} / \mathrm{N}$ ratio as demonstrated by its intense Raman peaks relative to the background noise. Power measurements were made at the sample surface using a handheld laser power meter to 
supplement instrumentation readings. This handheld device was used to ensure the power stayed well below $10 \mathrm{~mW}$.

The intensity of the resulting spectrum overall can vary due to how well the microscope focuses the incident laser on a fiber. As seen in Figure 6, the strand surface consists of areas of fibers at the surface (light) and areas of epoxy at the surface (dark). If the incident laser is focused directly on a fiber at the surface, the Raman peaks in the spectrum will be highly intense. However, if the laser is focused in between two fibers i.e. the darker areas, the peaks in the spectrum will appear lower in intensity. As a result, there is experimental variation in the intensity of spectra obtained from different areas of the same material, requiring some normalization of the spectrum characteristics as described in the following section.

\section{Spectrum Analysis}

Quantitative characterization of Raman spectra requires the specific characteristics of spectra be identified, such that spectra from different samples can be normalized and compared. A common method of comparing spectra is to analyze the shifting of individual peaks to either a higher or lower wavenumber. Changes in the peak position may be indicative of changes in structure of the material or stresses within the material $[6-12,24]$. For the present study, peak positions were analyzed as well as the bandwidth and the relative intensity of the peaks. This section describes the primary characteristics of the spectra examined in this study and how these characteristics were analyzed to compare spectra between strands with different histories of environmental exposure associated with the aging processes used.

The position of peaks within the Raman spectra was determined by Lorentzian curve fitting, using a best-fit curve with a Lorentzian fitting function. A Lorentzian fitting function is a continuous probability density function that is commonly used in spectroscopy for its ability to manipulate line shapes with scaled parameters. The process identifies the wavenumber of each peak in the spectrum that can be separated from the background noise, which is always present at some 
level. For Kevlar, there are between 8 and 16 individual peaks that can be identified in the range from 600 to $1800 \mathrm{~cm}^{-1}$, depending on the wavelength of the incident laser. Some of these peaks are quite low in intensity and therefore more difficult to locate on the spectrum consistently. To evaluate the shifting of peaks, the peak positions, i.e. absolute wavenumbers, were normalized by comparing the position of individual peaks with the peak located at $\sim 1613 \mathrm{~cm}^{-1}$ within a particular spectrum. Since each Raman peak stems from a different vibrational mode in the polymer, if a defect is present in the polymer chain, certain vibrational modes may be effected more than others. The normalized peak positions were calculated as follows:

$\Delta k_{i}=k_{i}-k_{1613}$

Where:

$k_{i}=$ Wavenumber of peak $i, \mathrm{~cm}^{-1}$

$\Delta k_{i}=$ Shifting peak $k_{i}$ relative to the 1613 peak, $\mathrm{cm}^{-1}$

$k_{1613}=$ Wavenumber of the $\sim 1613$ peak, $\mathrm{cm}^{-1}$

The position of the $\sim 1613 \mathrm{~cm}^{-1}$ peak can vary slightly from one spectrum to another, particularly when the instrument is recalibrated between measurements. In this case, the entire spectrum may be shifted, such that the absolute position of a particular peak is inadequate for analysis. The normalization of peak positions to the $\sim 1613 \mathrm{~cm}^{-1}$ peak within the spectrum allowed for the quantitative comparison of position.

A second characteristic of the spectra analyzed was the full width, half maximum (FWHM) of individual peaks within the spectrum. For this measurement, the width of the fitting function is determined at $1 / 2$ of the maximum intensity of the peak. This value is then normalized by determining the ratio of the individual peak's FHWM to the FHWM of the $\sim 1278 \mathrm{~cm}^{-1}$ peak for that spectrum:

$\eta_{i}=\frac{F W H M_{i}}{F W H M_{1278}}$ 
Where:

$\eta_{i}=\mathrm{FWHM}$ ratio (dimensionless)

$F W H M_{i}=\mathrm{FWHM}$ of peak $i, \mathrm{~cm}^{-1}$

$F W H M_{1278}=F W H M$ of $\sim 1278 \mathrm{~cm}^{-1}$ peak, $\mathrm{cm}^{-1}$

Finally, the relative intensity of the individual peaks was analyzed. Each intensity value was determined then normalized by calculating the ratio of the individual peak's intensity to the intensity of the $1278 \mathrm{~cm}^{-1}$ peak for that spectrum:

$\chi_{i}=\frac{I_{i}}{I_{1278}}$

Where:

$\chi_{i}=$ Intensity ratio (dimensionless)

$I_{i}=$ Intensity of peak $i, \mathrm{~cm}^{-1}$

$I_{1278}=$ Intensity for $\sim 1278 \mathrm{~cm}^{-1}$ peak, $\mathrm{cm}^{-1}$

\section{Mechanical Testing}

In addition to evaluating the effects elevated temperature and stress, the effect of applied loads on the Raman peak position was evaluated. Virgin Kevlar strands were placed in a miniature load frame with a cylindrical load cell designed to fit on the Raman microscope stage. Individual virgin Kevlar strands were mounted to tabs using a quick-setting epoxy to enable gripping of the strands by aluminum grips. Loading was applied in a staircase fashion (10 lbs steps) until failure. Raman spectra were obtained from the Kevlar strand at each load step using a $647 \mathrm{~nm}$ krypton laser with the beam polarized parallel to the fiber axis for all measurements. 


\section{Results}

\section{Comparison of Spectra}

Figure 9a indicates the Raman spectra at 647, 752 and 1064 nm, and Figure 9b indicates the Raman response at $488 \mathrm{~nm}$ for virgin Kevlar strands. The latter illustrates the increased background noise associated with fluorescence and specimen heating at shorter wavelengths such as the $488 \mathrm{~nm}$ laser. As shown in the figure, clear Raman spectra can be obtained at each incident wavelength, although the spectrum is saturated with fluorescence for the $488 \mathrm{~nm}$ laser.

Significant problems arise in obtaining a Raman spectrum due to fluorescence for most polymers when using a $488 \mathrm{~nm}$ argon laser. Fluorescence is a luminescence in which the molecular absorption of a photon triggers the emission of another photon with a longer wavelength. This photon is then shifted relative to the incident laser light, and appears at the same wavelengths as the Raman scattered photons. This change in energy between the absorbed photon and emitted photon transforms as molecular vibrations or heat. It is possible to avoid fluorescence problems by using a longer wavelength incident laser that sets the excitation frequency below the fluorescent envelope[15]. In this study, incident laser wavelength of 647, 752 and $1064 \mathrm{~nm}$ were used and found to successfully avoid these fluorescence effects.

Analytical peaks were previously calculated for Kevlar. Penn and Milanovich [16] used Raman spectra of model monomeric compounds and known structure-absorption correlations to obtain Raman band assignments for Kevlar. Kim et al. [17] used two model compounds to illustrate the different environments associated with two different benzene ring and amide groups of the chemical repeat unit in Kevlar. These models were used to calculate all vibrational modes of Kevlar using vibrational spectroscopy. The Raman band assignments by Penn and Milanovich correlated well with calculated values of Kim et al. The assignments determined by Penn and Milanovich and Kim et al. are shown in Table 2. 
Spectra obtained were compared to the analytical models in the literature to evaluate the effectiveness of the experimental processes, and identify any difference between the predicted peaks and those found experimentally. In general, there was good agreement between the experimental spectra and the predicted spectra. As shown in Table 2, experimental peak values could be related to 13 out of 14 of Penn and Milanovich analytical peaks, and 14 out of 15 for Kim et al. analytical peaks using an incident laser wavelength of $752 \mathrm{~nm}$. Using the FT Raman system with an incident laser wavelength of $1064 \mathrm{~nm}$, all theoretically predicted peaks could be identified. There was slightly less agreement using an incident wavelength of $647 \mathrm{~nm}$, finding 13 out 14 peaks predicted by Penn and Milanovich and 13 out 15 of the peaks predicted by Kim et al. It appears that the $752 \mathrm{~nm}$ laser has a lower $\mathrm{S} / \mathrm{N}$ ratio when compared to the $647 \mathrm{~nm}$ and $1064 \mathrm{~nm}$ lasers. There was significantly less agreement to the literature with an incident laser of $488 \mathrm{~nm}$ due to the fluorescence that saturates the spectrum. For shorter wavelengths, small peaks are more difficult to identify and may be lost in the background noise evident in Figure 9b, and as a

result these peaks are not identified. It is interesting to note a peak was found at $\sim 915 \mathrm{~cm}^{-1}$ using the 647,752 and $1064 \mathrm{~nm}$ lasers, but was absent in the literature. This peak was found in the Kevlar yarn as well as strand, eliminating the possibility that this is a resin effect. This peak was not identified in using an incident wavelength of $488 \mathrm{~nm}$. This $\sim 915 \mathrm{~cm}^{-1}$ peak was relatively low in intensity, and is likely not apparent due to the low $\mathrm{S} / \mathrm{N}$ ratio using the short wavelength laser.

\section{Effects of Aging}

Constant stress and elevated temperatures were used to "age" the Kevlar 49 specimens. Incident lasers used to detect aging effects were at $647 \mathrm{~nm}$ and $1064 \mathrm{~nm}$. The $752 \mathrm{~nm}$ laser was not used for the investigation of aging effects due to the low $\mathrm{S} / \mathrm{N}$ ratio as seen in Figure 9a.

Shifting of the peaks within the spectrum can be expected as a result of strain on the Kevlar fibers [6, 8-13]. For the aged specimens studied, which were previously stressed then tested without externally applied load, no significant peak shifting was detected relative to the $\sim 1613 \mathrm{~cm}^{-1}$ peak. 
It has been found that there are variations in the locations of peaks when using different wavelengths of incident light, but this is believed to be a measurement artifact.

The full width at half maxima (FWHM) was analyzed for each major Raman band for the virgin, creep tested, SIM, and the fleet leader samples. Figure 10 shows the FWHM ratio, $\eta_{i}$, on the vertical axis for each of the individual bands analyzed using the $647 \mathrm{~nm}$ incident laser for the creep specimens compared to the virgin specimen. From the figure, each data point represents an average of 3-5 measurements and illustrates the results for each of the Raman bands that were analyzed. Particular attention is given to the $\sim 1613 \mathrm{~cm}^{-1}$ band that is related to the ring vibrations of the $\mathrm{C}-\mathrm{C}$ phenyl ring and provides the backbone of the Kevlar microstructure. The FWHM ratio for the creep and fleet leader specimens are not consistent for most of the bands, with the exception of the 1613 and $1649 \mathrm{~cm}^{-1}$ band, in the spectrum relative to the virgin, unaged specimen tested. Figure 11 shows a closer view of the $\sim 1613 \mathrm{~cm}^{-1}$ band with the range of FWHM values for the virgin specimen and the creep_2F12j specimen. The high and low or "range" values are each single measurements of the virgin specimen and one random creep tested specimen. The average FWHM ratios for other creep specimens are also included in Figure 11.

In contrast to the creep and fleet leader samples, the FWHM of the SIM samples appeared to slightly decrease. This decrease may be due in part difficulties in measuring failed samples that have a low thickness relative to virgin samples used for comparison. These samples were also fractured during SIM testing, resulting in a frayed appearance. Proper orientation within the test apparatus was difficult to achieve.

Intensity analysis was performed for virgin, creep, SIM and the fleet leader samples using a 647 $\mathrm{nm}$ laser and a FT setup with a $1064 \mathrm{~nm}$ laser. The relative intensity of each band was compared with the relative intensity of bands from virgin specimens. Figure 12 shows the comparison of the relative intensities, $\chi_{i}$, using the incident laser of the $647 \mathrm{~nm}$ for the fleet leader and virgin specimens. The results shown in this figure indicate that there was a reduction in the relative 
intensity of the $1613 \mathrm{~cm}^{-1}$ band. Figure 13 shows a closer view of the $\sim 1613 \mathrm{~cm}^{-1}$ band with the range of intensity values for the virgin specimen and the SN 032a fleet leader specimen. The high and low or "range" values are each single measurements of the virgin specimen and one random fleet leader specimen. In addition, the average FWHM ratios for other fleet leader specimens are included in Figure 13. For the balance of the bands analyzed, there were mixed results when compared with the virgin specimens. The effect appears more pronounced for specimens SN032a and SN032b, which were sectioned from a tank that had been aged at an elevated temperature of $80^{\circ} \mathrm{C}$, up to $\chi_{1613} \approx 0.16$ for one of the specimens. Significant reduction was observed for the SIM and creep specimens as well.

\section{Effects of Applied Load}

The entire spectrum between 1100 and $1700 \mathrm{~cm}^{-1}$ shifted to lower wavenumbers under applied tensile stress for a virgin Kevlar composite strand. The $1613 \mathrm{~cm}^{-1}$ band showed the highest sensitivity with applied strain which is in direct agreement with the literature [14, 22]. The 1613 $\mathrm{cm}^{-1}$ band decreased $\sim 1.5 \mathrm{~cm}^{-1}$ per $10 \mathrm{lbs}$ of force. Figure 14 shows the decrease in wavenumber of the $1613 \mathrm{~cm}^{-1}$ band with applied loading. A line of best-fit has been inserted into the figure. The composite strand failed at $\sim 59 \mathrm{lbs}$ of applied tensile force. Another Kevlar composite strand was loaded and unloaded in a staircase fashion. When the strand was completely unloaded, the peak resorted back to the $1613 \mathrm{~cm}^{-1}$ position, concluding that the Kevlar fibers behaved elastically under the applied loading.

\section{Conclusions and Discussion}

The goal of this study was to develop and understand the Raman spectrum of Kevlar materials to provide a foundation for the development of NDE technologies based on laser light interaction with the Kevlar polymer. This has been examined by studying Raman spectra of four incident laser frequencies, correct Raman testing procedures, and effects of aging at increased temperature and stress of the Raman spectra of Kevlar composite materials. The incident laser 
frequency that provided the most suitable Raman spectra for analysis was the $647 \mathrm{~nm}$ laser. The $488 \mathrm{~nm}$ laser produced spectra that were saturated with fluorescence. At this frequency the Raman peaks are not well-defined due to the impossibility of removing fluorescence; therefore certain peaks could not be detected. For example, the $632,698,734$ and $915 \mathrm{~cm}^{-1}$ peaks were not detected at the $488 \mathrm{~nm}$ incident laser. The $752 \mathrm{~nm}$ laser experienced low fluorescence effects but had a low $\mathrm{S} / \mathrm{N}$ ratio. This problem may arise from the mechanical alignment in the spectrometer. The $1064 \mathrm{~nm}$ laser uses a Fourier transform system that requires a small sample size which is difficult to manipulate e.g. stress or strain. Another disadvantage using a long wavelength laser is the decreasing sensitivity of the Raman scattering effect. Changes in the spectra become less sensitive due to little reaction in the large cross section of the incident light compared to the small cross section of Raman scattering [15].

Testing reveals that it would be prudent to perform Raman measurements with the incident light polarized parallel to the polymer axis and the power kept under $10 \mathrm{~mW}$. The laser was polarized parallel to the polymer axis to create the strongest Raman scattering effect, and the power was kept under $10 \mathrm{~mW}$ to ensure no damage to the Kevlar specimen.

It has been observed that aged strand materials from fleet leader, SIM and creep specimens have decreased normalized intensity ratios $\left(\eta_{i}\right)$ for the $1613 \mathrm{~cm}^{-1}$ band when compared to unaged, virgin specimens. It has also been observed that the FWHM ratios $\left(\chi_{i}\right)$ have increased for the fleet leader and creep specimens when compared with virgin strand specimens. In other words, the peaks are generally broader and of lower intensity than for virgin, undamaged specimens. Increases in FWHM may be attributed to defects in the fiber or defects being created in the fiber under stress [6]. On a molecular level, rod-like crystallites within the Kevlar distribute loading through hydrogen bonds between adjacent crystallites. Damage to crystallites due to aging phenomena may result in a redistribution of stresses on a molecular level, as damaged crystallites shed loading. As a result, variations in stresses between crystallites exist, leading to a broader sprectrum of scattering. 
It was also observed that peak position was invariant as a result of exposure to increased stress and temperature. This is significant in that there is substantial literature to suggest that peak shifting as a result of applied strain can be used experimentally to determine loading of Kevlar fibers $[6,9,12]$. The observation that these peak positions are unaffected by this "aging" suggests that the relationship between peak position and applied strain could be applied without regard to the "age" of the material. Therefore, evaluation of peak position could form one component of an NDE technique that compared the applied loads in-situ with loading estimated by theoretical models, to locate areas of stress concentration or to analyze the effects of creep. As reported, the entire spectrum shifted due to applied load.

Raman spectroscopy has been successfully applied to Kevlar composite materials to gain an understanding of how the spectra changes with different experimental conditions. A foundation has been created for further investigation into environmental degradation of Kevlar materials using Raman spectroscopy.

\section{References}

1. Gerstle, F.P. Prediction of Long-term Failure in Kevlar 49 Composites. in SAND-812072C; CONF-820340-2. 1982. Sandia National Laboratories - Albuquerque, NM.

2. Mertz, D.R., Chajes, M.J., Gillespie, J.W., Kukich, D.S., Sabol, S.A., Hawkins, N.M., Aquino, W., Deen, T.B., Application of Fiber Reinforced Polymer Composites to the Highway Infrastructure, in NCHRP Report 503. 2003: Wasington D.C.

3. Washer, G., Allempalli, S. NDE Technologies for Condition Assessment of FRP Retrofits. in ASNT Fall Conference. 2005. Columbus, OH.

4. Duke, J.C., Horne, M.R. NDE of Polymeric Composite Material Bridge Components. in The International Society for Optical Engineering. 1998.

5. Karbhari, V.M., Chin, J.W., Hunston, D., Benmokrane, B., Juska, T., Morgan, R., Lesko, J.J., Sorathia, U., Reynaud, D., Durability Gap Analysis for Fiber-Reinforced Polymer Composites in Civil Infrastructure. Journal of Composites for Construction, 2003(August): p. 238-247.

6. Prasad, K., Grubb, D.T., Deformation Behavior of Kevlar Fibers Studied by Raman Spectroscopy. Journal of Applied Polymer Science, 1990. 41: p. 2189-2198.

7. Andrews, M.C., Bannister, D.J., Young, R.J., The Interfacial Properties of Aramid/Epoxy Model Composites. Journal of Materials Science, 1996. 31: p. 3893-3913. 
8. Chang, C., Hsu, S. L., An Analysis of Strain-Induced Frequency Changes in Poly ( $p$ phenylene terephthalamide) Single Fibers. Macromolecules, 1990. 23: p. 1484-1486.

9. Galiotis, C., Robinson, I.M., Young, R.J., Smith, B., Batchelder, D. N., Strain Dependence of the Raman Frequencies of a Kevlar 49 Fiber. Polymer Communications, 1985. 26: p. 354-355.

10. Kawagoe, M., Hashimoto, S., Nomiya, M., Morita, M., Qiu, J., Mizuno, W., Kitano, H., Effect of Water Absorption and Desorption on the Inerfacial Degradation in a Model Composite of an Aramid Fiber and Unsaturated Polyester Evaluated by Raman and FT Infra-red Microscopy. Journal of Raman Spectroscopy, 1999. 30: p. 913-918.

11. Parthenios, J., Katerelos, D. G., Psarras, G.C., Galiotis, C., Aramid fibers; a multifunctional sensor for monitoring stress/strain fields and damage development in composite materials. Engineering Fracture Mechanics, 2002. 69: p. 1067-1087.

12. Schadler, L.S., Galiotis, C., Fundamentals and Applications of Micro Raman Spectroscopy to Strain Measurements in Fiber Reinforced Composites. International Materials Reviews, 1995. 40(3): p. 116-133.

13. Young, R.J., Day, R.J., Zakikhani, The Structure and Deformation Behavior of poly ( $p$ phenylene benzobisoxazole) Fibers. 1990: p. 127-136.

14. Ferraro, J.R., Nakamoto, K., Introductory Raman Spectroscopy. 1994, San Diego: Academic Press, Inc.

15. Koenig, J.L., Spectroscopy of Polymers. Koenig, J.L. 1992, Washington, DC: ACS Professional Reference Book.

16. Penn, L., Milanovich, F., Raman Spectroscopy of Kevlar 49 Fiber. Polymer, 1979. 20(1): p. 31-36.

17. Kim, P.K., Chang, C., Hsu, S.L., Normal Vibrational Analysis of a Rigid Rod Polymer: poly(p-phenylene terephthalamide). Polymer, 1986. 27(1): p. 34-46.

18. Stuart, B.H., A Fourier Transform Raman Study of Water Sorption by Kevlar-49. Polymer Bulletin, 1995. 35: p. 727-733.

19. Yang, H.H., Kevlar Aramid Fiber. 1993, New York: Wiley.

20. Alwis, K.G.N.C., Burgoyne, C. J. Stepped Isothermal Method for Creep Rupture Studies of Aramid Fibers. in ACI Special Publications. 2005.

21. Chase, D.B., Rabolt, J. F., ed. Fourier Transform Raman Spectroscopy: From Concept to Experiment. Fourier Transform Raman Spectroscopy, ed. D.B. Chase, Rabolt, J. F. 1994, Academic Press Inc.: San Diego.

22. Hallmark, V.M., ed. FT - Raman Spectroscopy as a Structural Probe of Polymers and Other Long-Chain Organic Molecules. Fourier Transform Raman Spectroscopy, ed. D.B. Chase, Rabolt, J. F. 1994, Academic Press, Inc.: San Diego.

23. Galiotis, C., Robinson, I.M., Young, R.J., Batchelder, D. N., ed. Strain Measurements in Aramid Fibers and Composites Using Raman Spectroscopy. Engineering Applications of New Composites. 1988.

24. Young, R.J., Day, R.J., Lu, D., Raman Spectroscopy of Kevlar Fibers during Deformation - Caveat Emptor. Polymer International, 1991. 24: p. 71-76. 
Table 1. Raman scattering analyses of Kevlar have determined a series of well defined peaks ranging from 600 to $1700 \mathrm{~cm}^{-1}$ as shown in Table 1 .

Table 2. List of theoretical peaks compared to the experimental peaks.

\begin{tabular}{|l|l|}
\hline Wavenumber $\left(\mathbf{c m}^{-1} \mathbf{)}\right.$ & Assignment \\
\hline $630,732,786$ & Ring vibrations \\
\hline 845 & C-H out-of-plane bending \\
\hline 863 & Ring vibrations \\
\hline 1103 & C-H in-plane bending \\
\hline $1181,1277,1327,1514$ & C-C ring stretching \\
\hline 1318 & C-H in-plane bending \\
\hline 1569 & Amide II $60 \%$ N-H bending; 40\% C-N stretching \\
\hline 1610 & C-C ring stretching \\
\hline 1648 & $\begin{array}{l}\text { Amide I (80\% C=O stretching; } 10 \% \text { C-N } \\
\text { stretching } 10 \% \text { N-H bending) }\end{array}$ \\
\hline
\end{tabular}

\begin{tabular}{|c|c|c|c|c|c|c|}
\hline \multicolumn{7}{|c|}{ RAMAN PEAKS $\left(\mathrm{cm}^{-1}\right)$} \\
\hline \multicolumn{7}{|c|}{ TEST TYPE } \\
\hline \multicolumn{2}{|c|}{ Theoretical } & \multirow{2}{*}{$\begin{array}{r}\text { Kevlar yarn } \\
(647 \mathrm{~nm})\end{array}$} & \multicolumn{4}{|c|}{ Kevlar strand } \\
\hline $\begin{array}{l}\text { Penn and } \\
\text { Milanovich } \\
\text { Peaks }\end{array}$ & $\begin{array}{c}\text { Kim et al. } \\
\text { Peaks }\end{array}$ & & $(488 \mathrm{~nm})$ & $(647 \mathrm{~nm})$ & (752 nm) & (1064 nm) \\
\hline 632 & 637 & & & 631 & 630 & 630 \\
\hline 698 & 694 & & & 700 & 697 & 695 \\
\hline 734 & 725 & & & 731 & 732 & 732 \\
\hline 789 & 773 & 790 & 784 & 789 & 787 & 787 \\
\hline- & 853 & & & & 847 & 845 \\
\hline - & - & 914 & & 915 & 913 & 918 \\
\hline 1104 & 1106 & 1105 & & 1105 & 1105 & 1108 \\
\hline 1187 & 1187 & 1184 & 1186 & 1184 & 1185 & 1186 \\
\hline 1192 & 1188 & & & & & 1194 \\
\hline 1279 & 1283 & 1280 & 1278 & 1280 & 1278 & 1283 \\
\hline 1331 & 1332 & 1331 & 1331 & 1330 & 1329 & 1332 \\
\hline 1409 & 1400 & 1413 & & 1411 & 1415 & 1417 \\
\hline 1518 & 1516 & 1519 & 1520 & 1518 & 1517 & 1521 \\
\hline 1570 & 1567 & 1572 & 1577 & 1571 & 1570 & 1574 \\
\hline 1615 & 1615 & 1613 & 1612 & 1613 & 1612 & 1615 \\
\hline 1649 & 1654 & 1649 & 1649 & 1649 & 1649 & 1651 \\
\hline
\end{tabular}


Figure 1. Raman scattered photons are shifted from the incident energy due to change from the vibrational energy of the molecule.

Figure 2. Schematic diagram of a Raman spectrometer system.

Figure 3. Raman spectrum of Kevlar illustrating the wavenumber conversion process.

Figure 4. The chemical structure of Kevlar.

Figure 5. Raman spectrum of Kevlar. The vibrational modes of the 1613 and $1651 \mathrm{~cm}^{-1}$ bands are indicated.

Figure 6. Two views of a Kevlar strand rotated at different angles under the Raman microscope.

Figure 7. Differences in appearance of the spectrum when the laser is polarized parallel to the fiber axis and perpendicular to the fiber axis.

Figure 8. Raman spectrum of Kevlar at different power levels.

Figure 9. Raman spectra of Kevlar using an incident laser of (a) 647, 752 and $1064 \mathrm{~nm}$ and (b) $488 \mathrm{~nm}$.

Figure 10. The FWHM ratio, $\eta_{i}$, using the $647 \mathrm{~nm}$ incident laser for the creep specimens compared to a virgin specimen.

Figure 11. Average values compared with the range for FWHM ratios of creep specimens compared to a virgin specimen.

Figure 12. Comparison of the relative intensities, $\chi_{i}$, using the incident laser of the $647 \mathrm{~nm}$ for the fleet leader and virgin specimens.

Figure 13. Average values compared with the range for intensity ratios of fleet leader specimens compared to a virgin specimen.

Figure 14. Decrease in wavenumber of the $1613 \mathrm{~cm}^{-1}$ band with applied tensile stress. A line of best-fit has been inserted into the figure. 

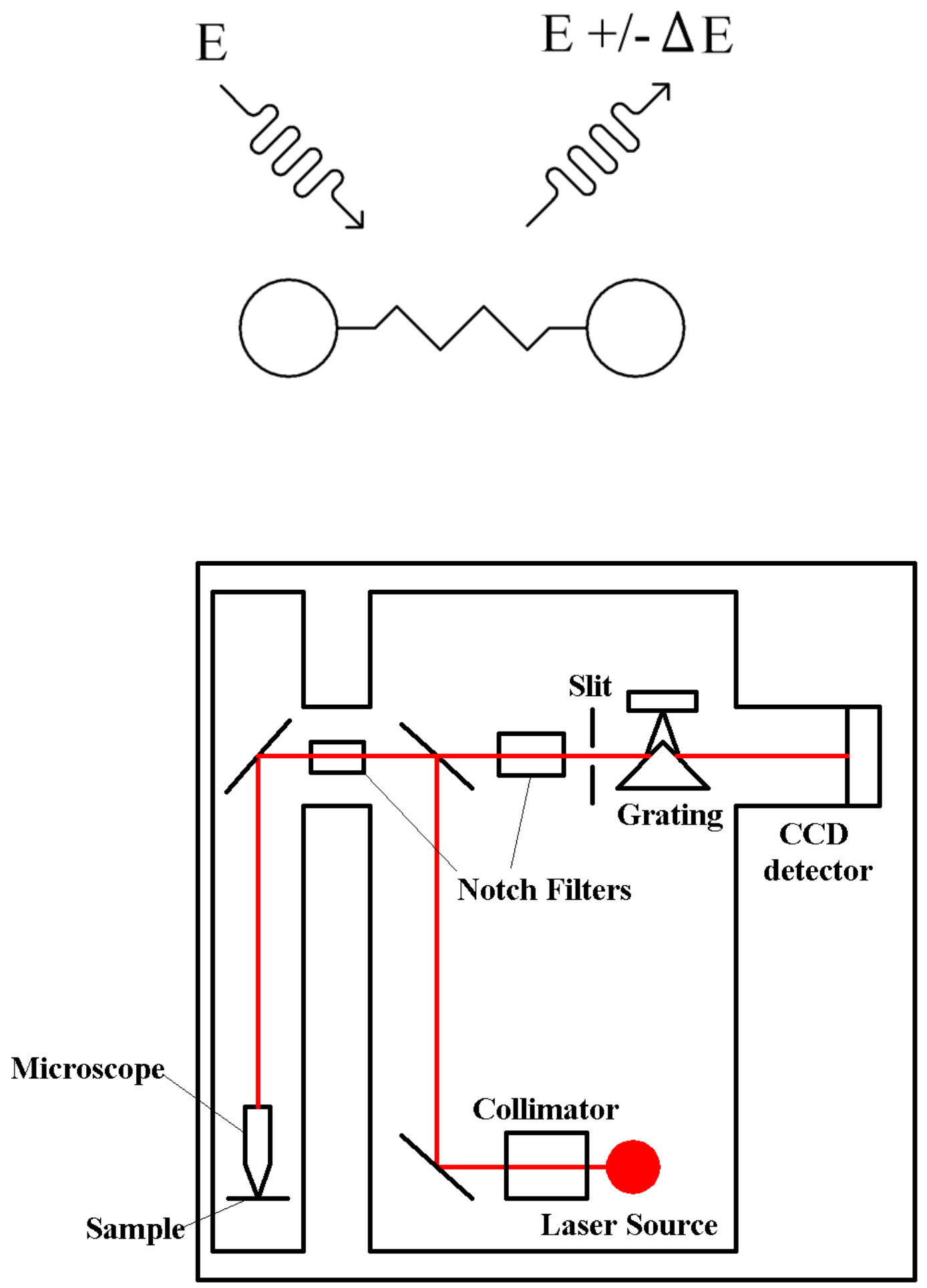


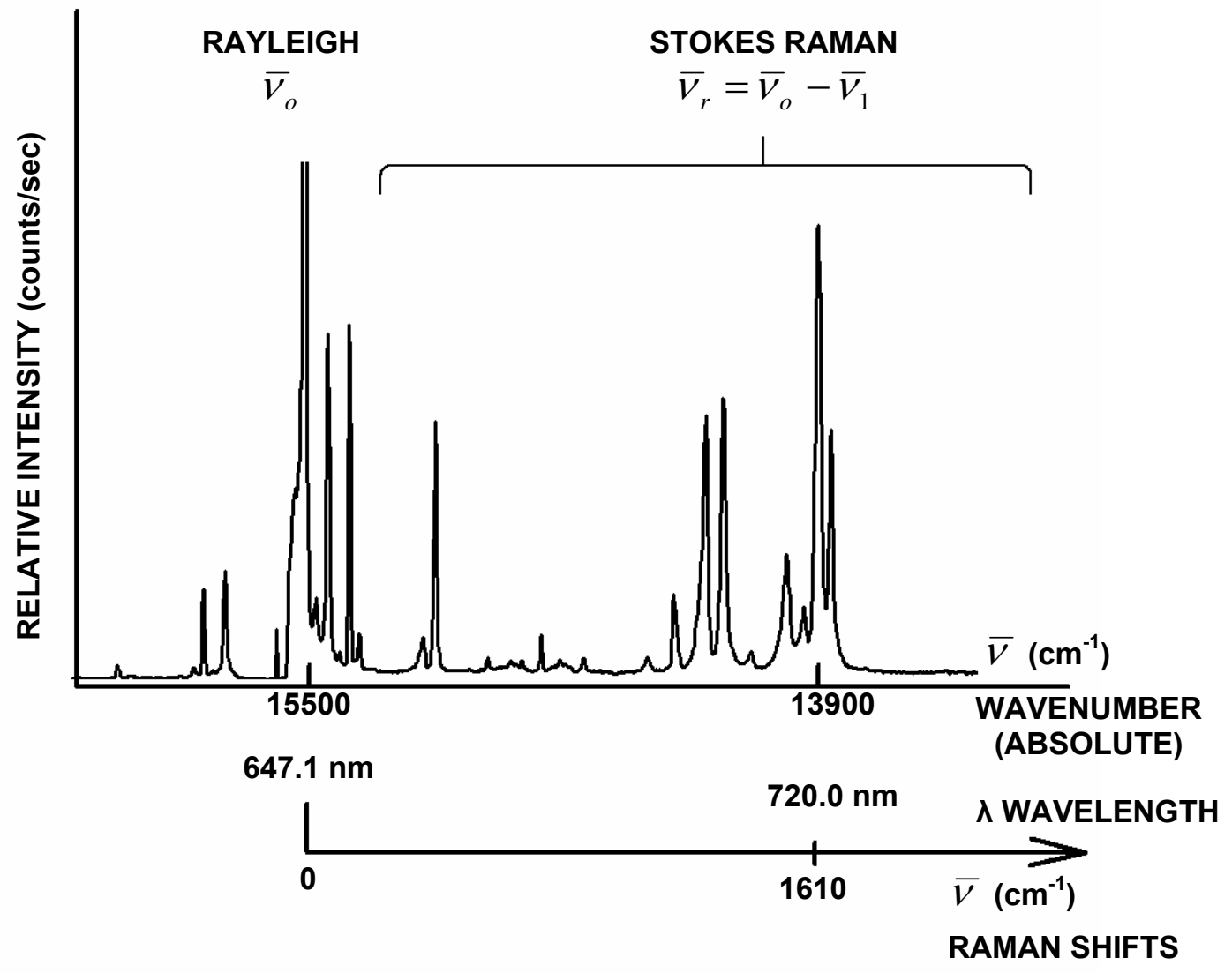



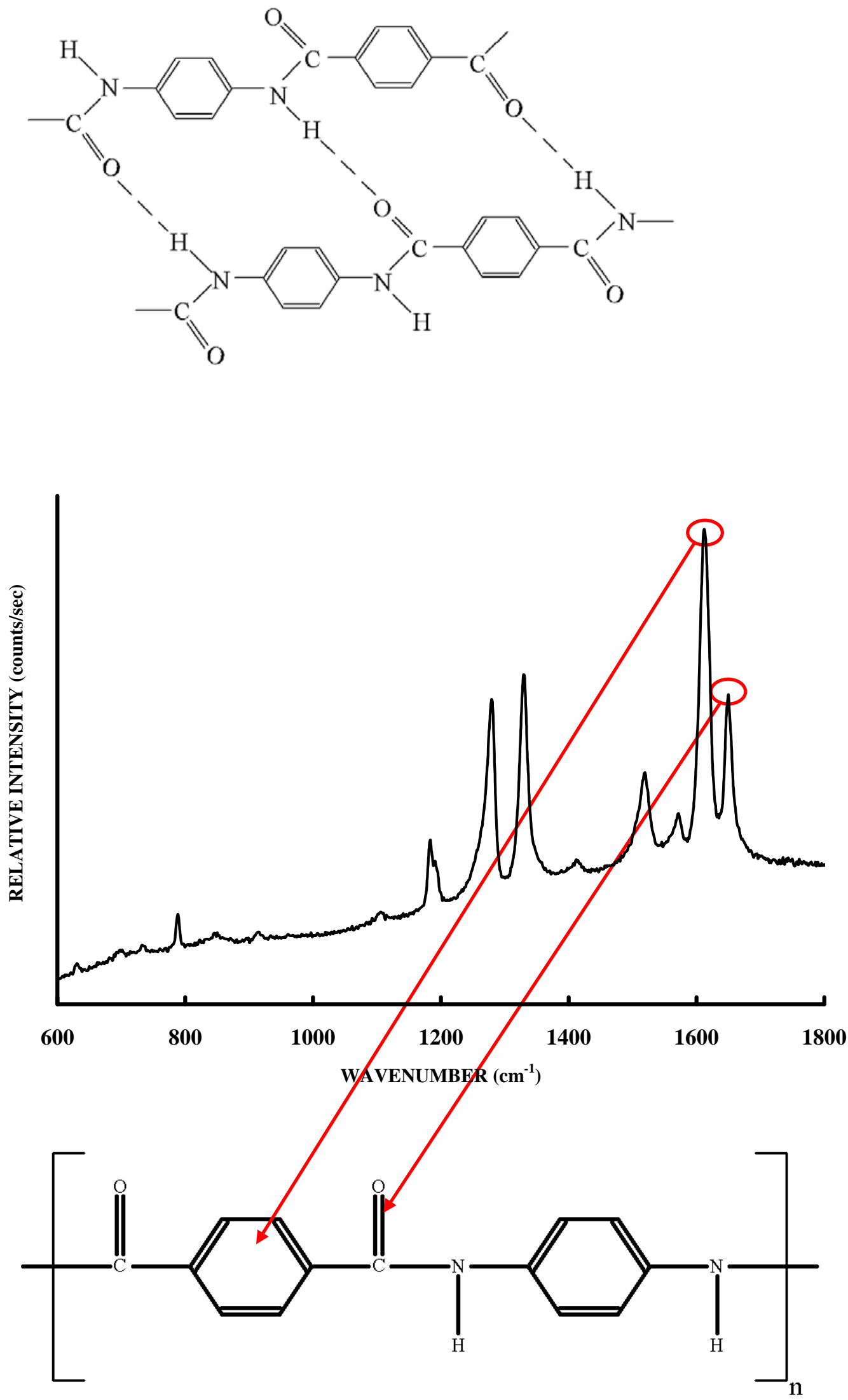

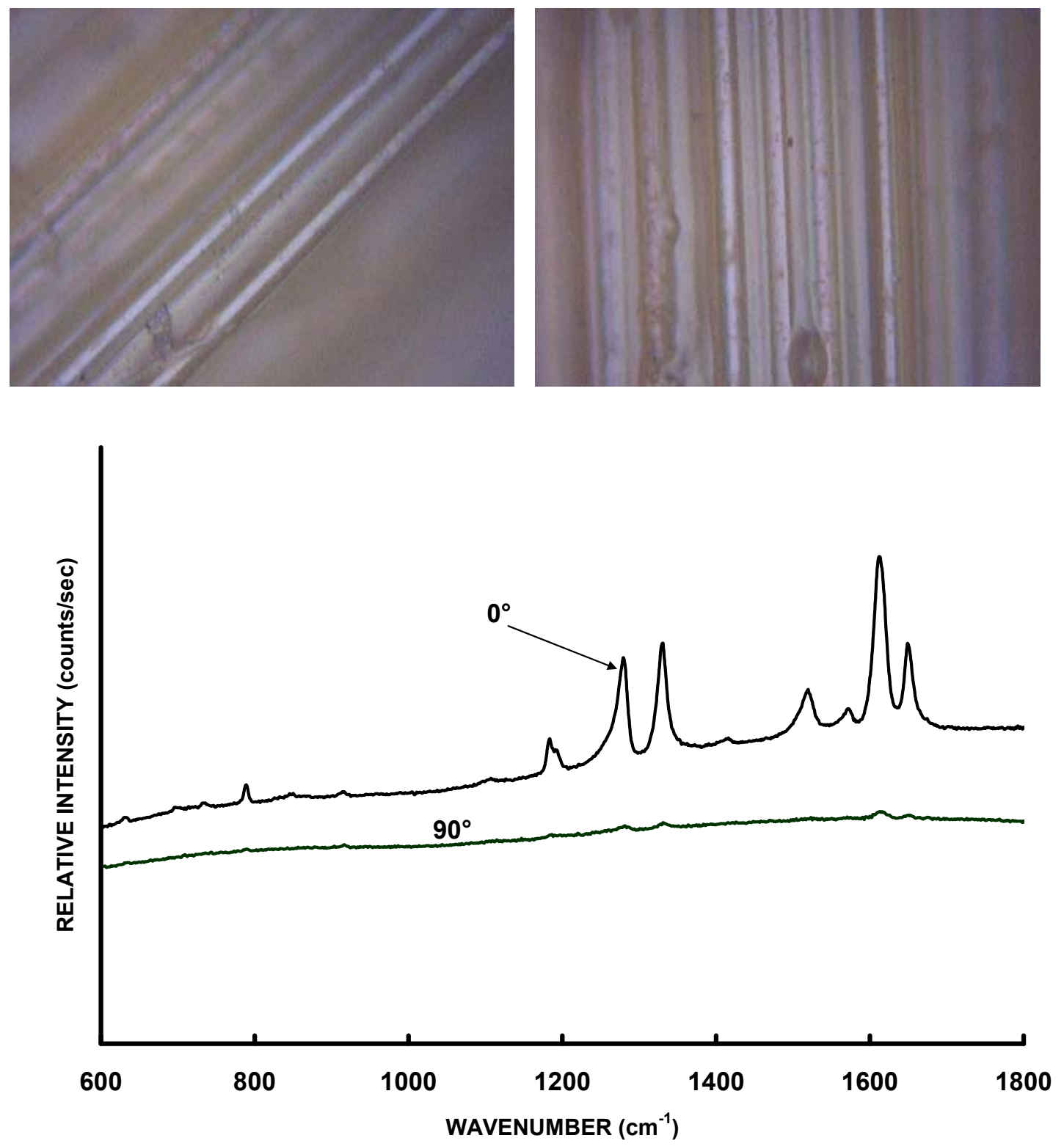

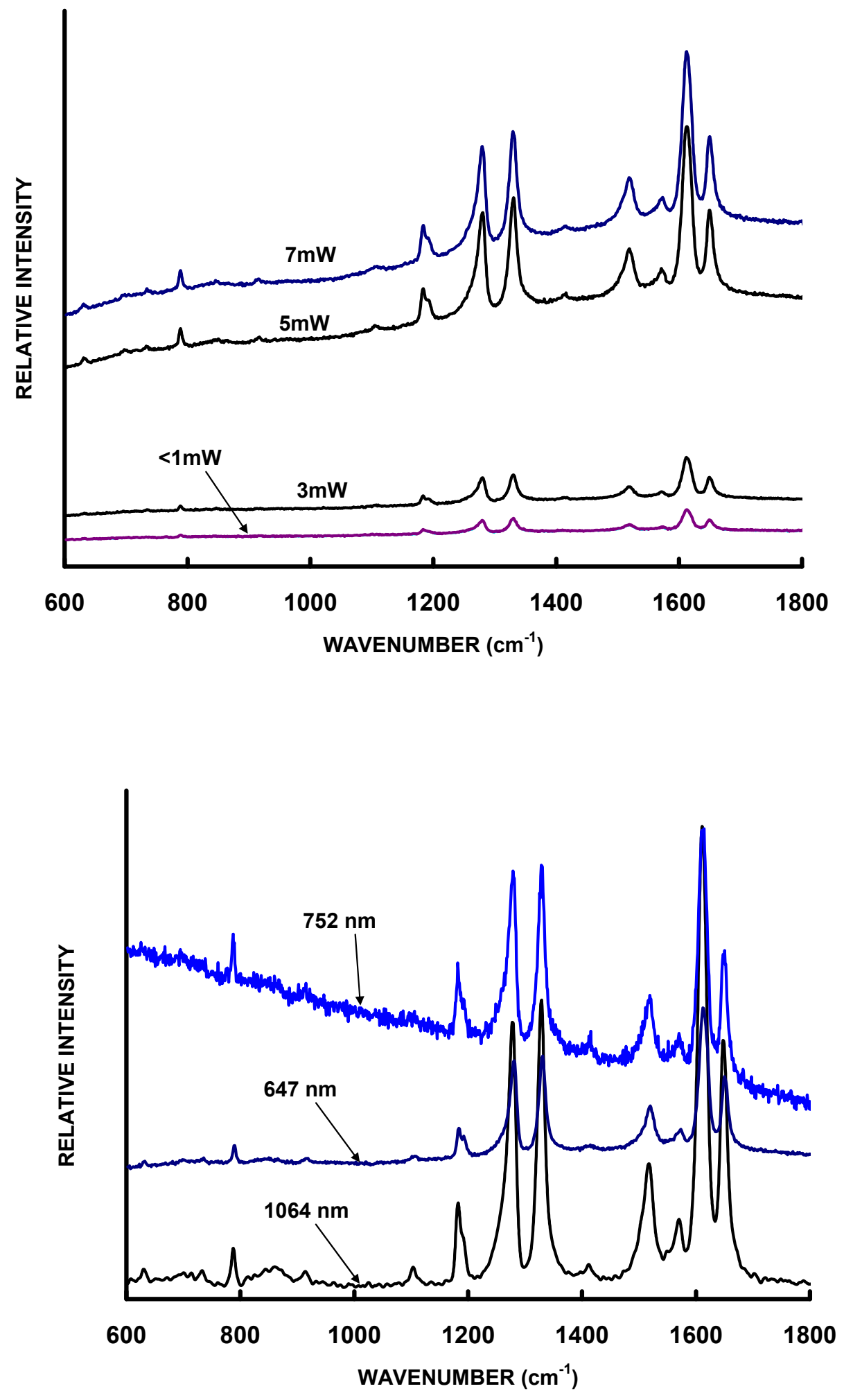

(a) 


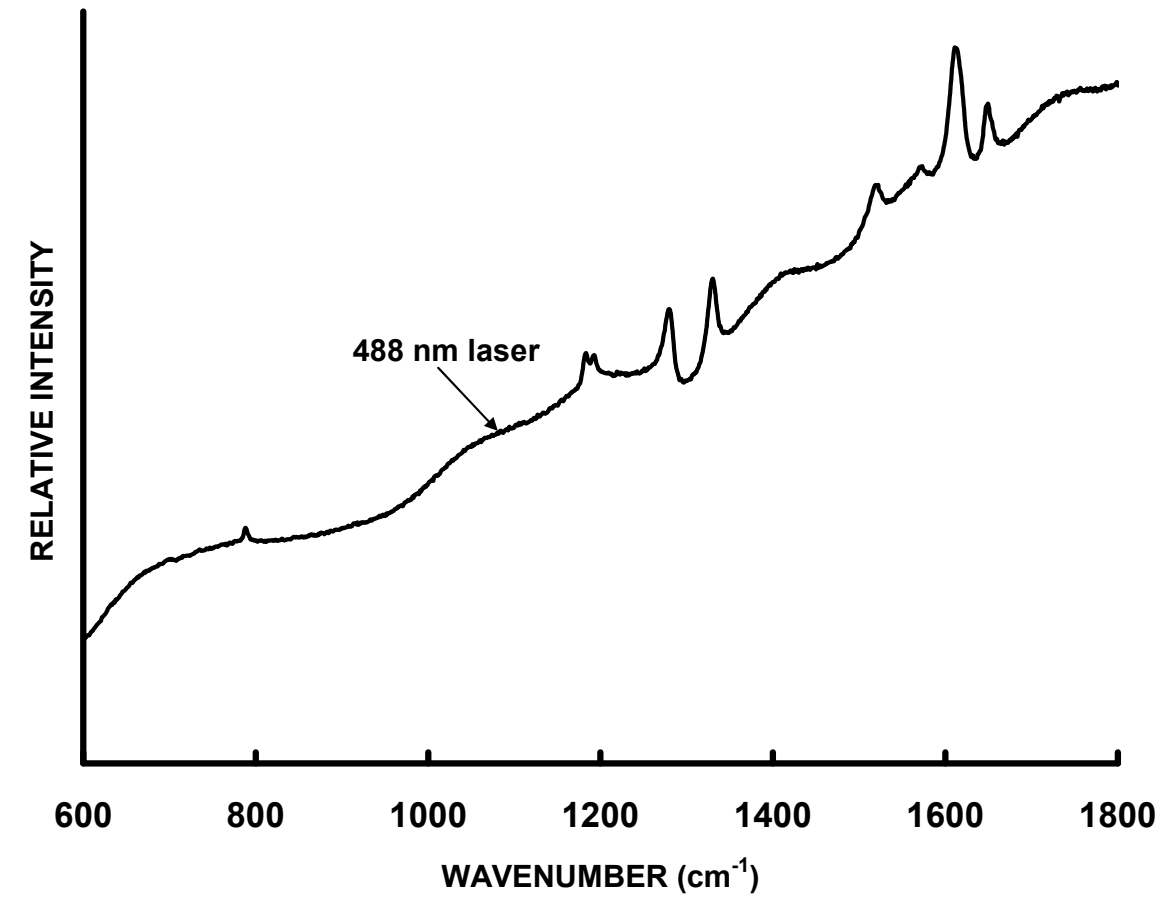

(b) 

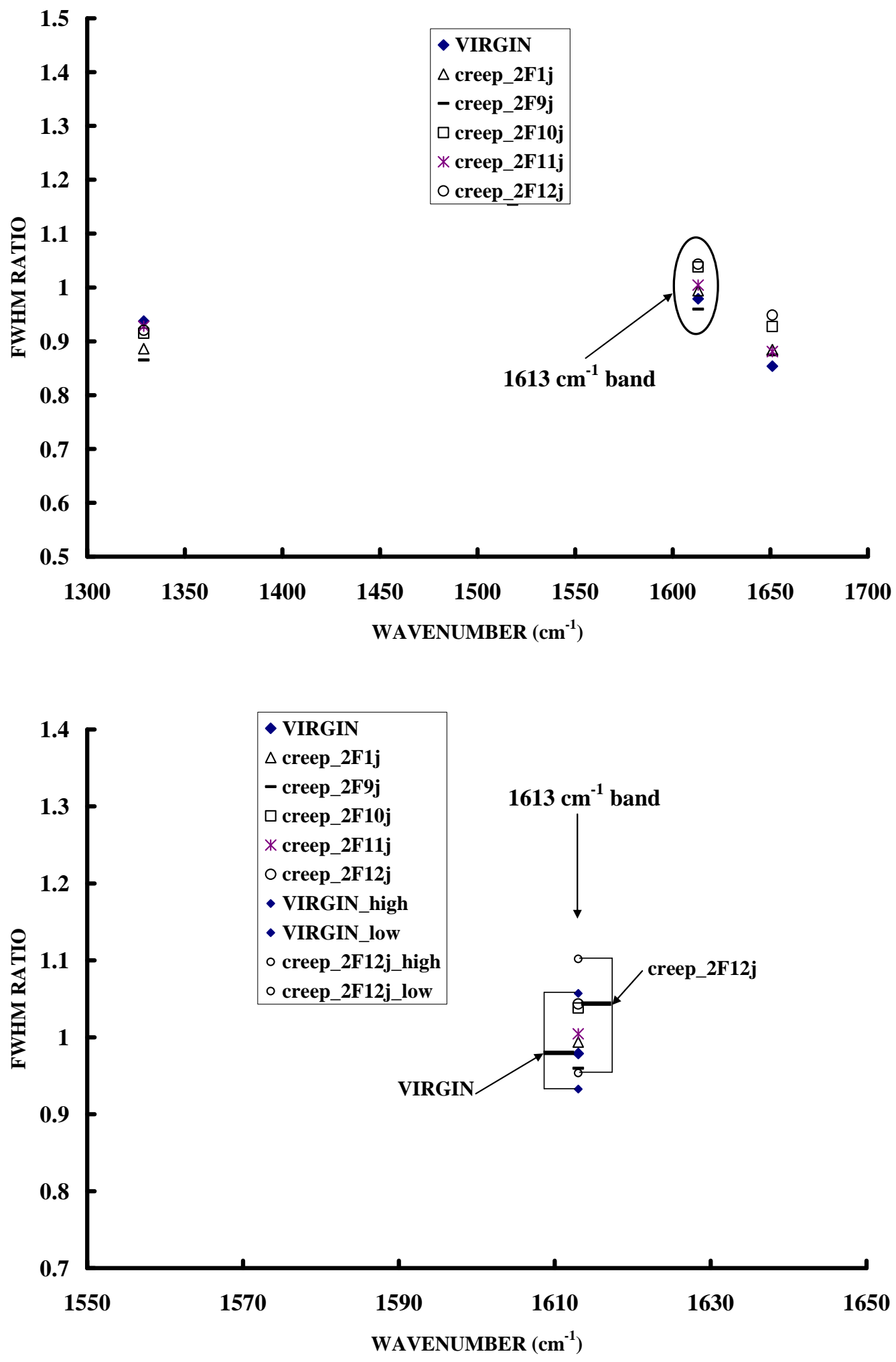

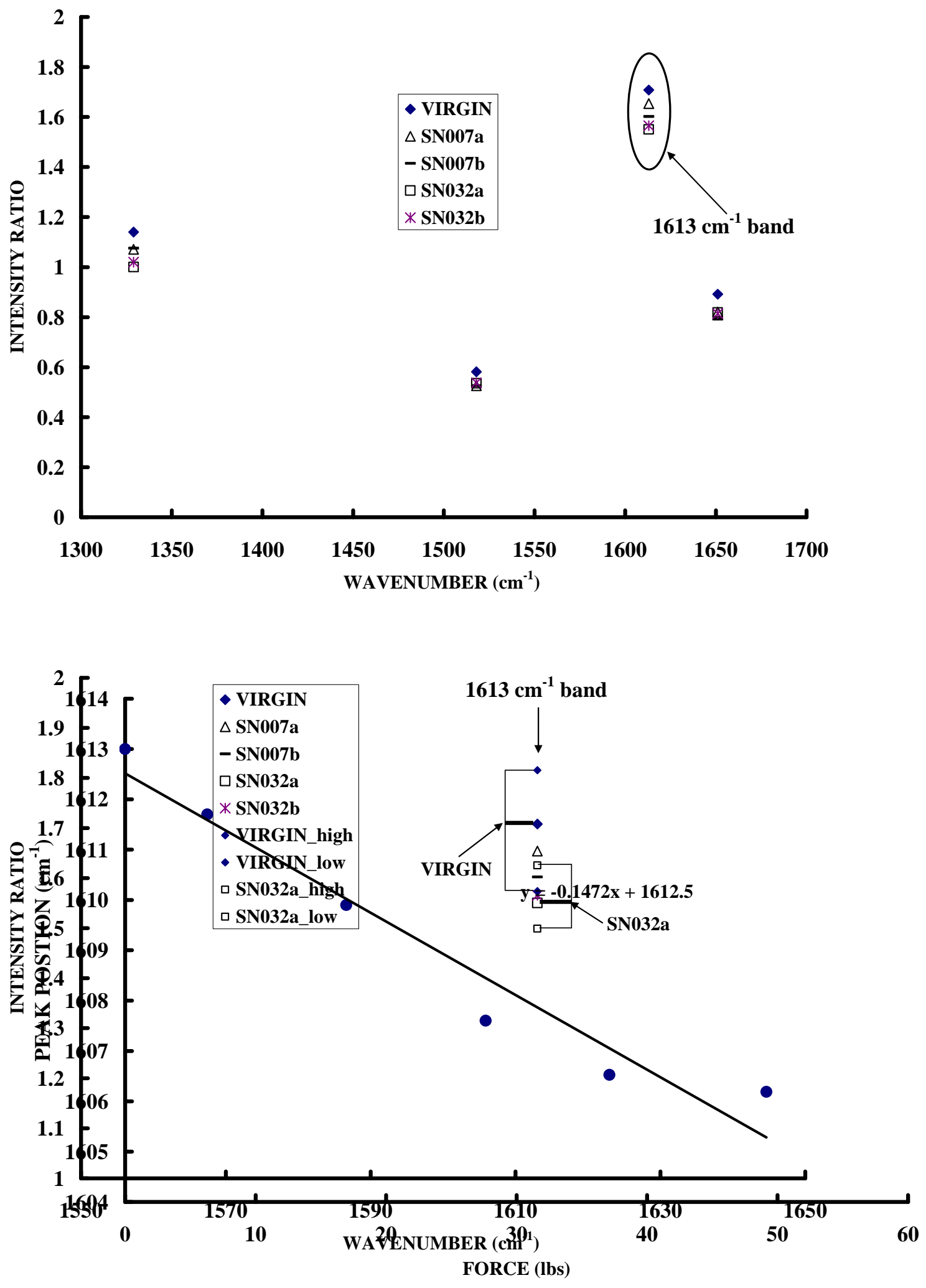